This document is the accepted manuscript version of the following article:

Krieger, M., Klimpki, G., Fattori, G., Hrbacek, J., Oxley, D., Safai, S., ... Zhang, Y. (2018). Experimental validation of a deforming grid 4D dose calculation for PBS proton therapy. Physics in Medicine and Biology, 63(5), 055005 (11 pp.).

https://doi .org/10.1088/1361-6560/aaad1e

\title{
Experimental validation of a deforming grid 4D dose calculation for PBS proton therapy
}

\author{
Miriam Krieger ${ }^{1,2}$, Grischa Klimpki ${ }^{1,2}$, Giovanni Fattori ${ }^{1}$, Jan \\ Hrbacek $^{1}$, David Oxley ${ }^{1}$, Sairos Safai ${ }^{1}$, Damien C. Weber ${ }^{1,3}$, \\ Antony J. Lomax ${ }^{1,2}$, Ye Zhang ${ }^{1}$ \\ ${ }^{1}$ Paul Scherrer Institute (PSI), Center for Proton Therapy, 5232 Villigen PSI, \\ Switzerland \\ 2 Department of Physics, ETH Zurich, 8092 Zurich, Switzerland \\ ${ }^{3}$ Department of Radiation Oncology, University Hospital Zurich, 8091 Zurich, \\ Switzerland \\ E-mail: miriam.krieger@psi.ch
}

\begin{abstract}
The aim of this study was to verify the temporal accuracy of the estimated dose distribution by a $4 \mathrm{D}$ dose calculation (4DDC) in comparison to measurements. A single-field plan (0.6Gy), optimised for a liver patient case (CTV volume: 403cc), was delivered to a homogeneous PMMA phantom and measured by a high resolution scintillating-CCD system at two water equivalent depths. Various motion scenarios (no motion and motions with amplitude of $10 \mathrm{~mm}$ and two periods: $3.7 \mathrm{~s}$ and $4.4 \mathrm{~s}$ ) were simulated using a 4D Quasar phantom and logged by an optical tracking system in real-time. Three motion mitigation approaches (single delivery, 6x layered and volumetric rescanning) were applied, resulting in 10 individual measurements. 4D dose distributions were retrospectively calculated in water by taking into account the delivery $\log$ files (retrospective) containing information on the actually delivered spot positions, fluences, and time stamps. Moreover, in order to evaluate the sensitivity of the $4 \mathrm{DDC}$ inputs, the corresponding prospective 4DDCs were performed as a comparison, using the estimated time stamps of the spot delivery and repeated periodical motion patterns. 2D gamma analyses and dose-difference-histograms were used to quantify the agreement between measurements and calculations for all pixels with $>5 \%$ of the maximum calculated dose. The results show that a mean gamma score of $99.2 \%$ with standard deviation $1.0 \%$ can be achieved for $3 \% / 3 \mathrm{~mm}$ criteria and all scenarios can reach a score of more than $95 \%$. The average area with more than $5 \%$ dose difference was $6.2 \%$. Deviations due to input uncertainties were obvious for single scan deliveries but could be smeared out once rescanning was applied. Thus, the deforming grid 4DDC has been demonstrated to be able to predict the complex patterns of $4 \mathrm{D}$ dose distributions for PBS proton therapy with high dosimetric and geometric accuracy, and it can be used as a valid clinical tool for $4 \mathrm{D}$ treatment planning, motion mitigation selection, and eventually 4D optimisation applications if the correct temporal information is available.
\end{abstract}

Keywords: proton therapy, 4D dose calculation, pencil beam scanning, liver tumour

Submitted to: Phys. Med. Biol. 


\section{Introduction}

In recent years, many proton therapy centres have been planned and built, with a majority of them delivering pencil beam scanning (PBS). However, there is a major challenge in PBS particle therapy, namely the handling of intra-fractional motion. Whereas for passive scattering, target coverage can in principle be conserved by the simple application of adequately defined treatment margins (both laterally and range adjusted), the so-called interplay effect resulting from the temporal dependence of the dose delivery for PBS leads to significant degradation of the dose distribution when delivered in the presence of motion (Phillips et al. (1992), Bert \& Durante (2011)). In addition, density changes in the beam path, e.g. due to motion of the ribs, lead to significant changes in proton range and thus to the depth of the main dose deposition for every pencil beam (Engelsman et al. (2013)). Several approaches have been suggested, and partly clinically implemented, to mitigate these effects (Groezinger et al. (2008), Knopf et al. (2010)). However, to estimate the effectiveness of these for a particular patient, a four dimensional dose calculation (4DDC) tool is useful to accurately estimate the time dependence of the delivery and the interaction of this with the dynamics of motion. Various approaches for such a tool are in use at different institutes (Bert \& Rietzel (2007) and Richter et al. (2013) (GSI), Ammazzalorso \& Jelen (2014) (University of Marburg), Paganetti et al. (2005) (MGH), Kang et al. (2005) and Li et al. (2014) (MD Anderson)) and have been recently incorporated into commercial treatment planning systems (Engwall et al. (2016) (Raystation, Stockholm, Sweden)).

An early form of 4DDC was described by Rietzel et al. (2005) who calculated the static dose distribution on every single phase of a 4DCT and then averaged the resulting distributions. The major disadvantage of this approach is the simplicity of ignoring the exact timing of the sequential beam delivery which assumes that the dose can be delivered homogeneously to all the CT phases, which is clearly not realistic for pencil beam scanning. A more advanced approach is to calculate the subplan of the static dose distribution on each phase of a 4DCT, depending on the timing of the spot delivery sequence, and then accumulate the dose on a single (reference) CT data set (Bert et al. (2008)). However, this approach is necessarily restricted to the temporal resolution of the 4DCT (typically of the order of $0.5 \mathrm{~s}$ ) and must assume that, within this time scale, all pencil beams are delivered on a static geometry. Experimental validations of such dose calculations have been performed by various groups, taking into account different uncertainties. Testa et al. (2013) comprehensively validated their Monte Carlo based dose calculation engine for passive scattering proton therapy, measuring depth-dose-curves and 2D dose profiles (using an array of ionisation chambers with spatial resolution: $7.62 \mathrm{~mm}$ ). The results were evaluated in terms of clinical tolerances. High-resolution dose measurements were performed by Bert et al. (2009, 2012) using radiographic films. Motion effects were extracted from the films and compared to previously simulated effects. The evaluation was done by quantifying relative dose differences and line profiles. More recently, Pfeiler et al. (2017) have used delivery 
$\log$ files to reconstruct accurate 4D dose distributions and have compared these to measurements performed using a moving phantom and ionisation chamber array.

In contrast, the 4DDC used at our institute is based on a deforming dose grid approach (see e.g. Boye et al. (2013), Bernatowicz et al. (2013), and Zhang et al. (2012)). Briefly put, instead of warping and accumulating the dose distribution on each image phase, our approach deforms the calculational dose grid as a function of time, the vectors for this deformation being extracted from 4D imaging such as 4DCT or 4DMRI (Boye et al). This approach allows to model motion effects at much higher temporal resolutions than the conventional approach, and many studies on the effects of motion and the effectiveness of motion mitigation strategies have been conducted using this 4D dose calculation (Bernatowicz et al. (2013), Bernatowicz et al. (2016), Knopf et al. (2011), Knopf et al. (2013), Dueck et al. (2016), Gorgisyan et al. (2017), Zhang et al. (2012), Zhang et al. (2013), Zhang et al. (2014), Zhang et al. (2015), Zhang et al. (2016), Zhang

et al. (2017)). As such, and as is the case for all other components of a treatment planning system, such a 4DDC needs to be commissioned and verified, particularly its ability to model the complex effects of the temporal interplays between the delivery dynamics of PBS proton therapy and motion of the patient.

It is the aim of this study therefore to experimentally validate the temporal accuracy of the deforming grid 4D dose calculation under well-defined experimental conditions and for different assumptions on the temporal dynamics of the delivery, different motion periods and with different motion mitigation approaches. For this, we use a high spatial resolution dosimetry system, as the work of Phillips et al. (1992) has shown that even small motions can cause dosimetric effects with dimensions smaller than that of the pencil beam spacing or beam width.

\section{Materials and methods}

A critical part of the $4 \mathrm{D}$ dose calculation process is knowledge of both the organ motion of the patient and the exact temporal dynamics of the PBS delivery system. As such, in this experimental validation, we have studied the accuracy of $4 \mathrm{D}$ dose calculations using the deforming grid approach under two assumptions about PBS delivery dynamics. First, that these are known as accurately as possible from log files of the delivered treatments (see e.g. Meier et al. (2015), Scandurra et al. (2016) and Belosi et al. (2017)). This approach is referred to in this work as a retrospective approach. Second, delivery dynamics are predicted using estimates of the reaction times of the scanning elements and beam intensities to be used for delivery (called prospective calculations). Such a prospective approach can be important for (e.g.) estimating motion effects on treatments a priori as part of a patient specific quality assurance procedure (Hrbacek et al, PTCOG 2017, Yokohama) or as input into a 4D optimisation paradigm (see e.g. Bernatowicz et al. (2017)). On the other hand, the retrospective (log-file based) approach is important for post-treatment reconstruction of the delivered 4D dose (Richter et al. (2014), Richter et al. (2013)). 


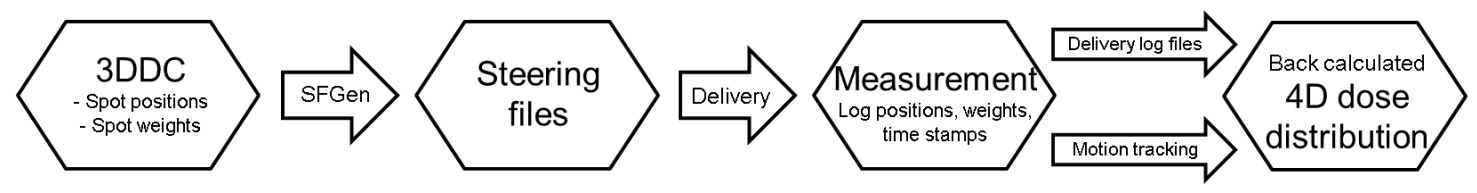

Figure 1: Illustration of the procedure applied to retroscpectively calculate the 4D dose distributions. As in clinical routine, a 3D plan with the corresponding spot list is created. The steering file generator (SFGen) converts the plan information into machine readable steering files which are used to deliver the plan. During the delivery, the dose distribution in one specific water equivalent depth is measured and the actual spot positions, weights, and time stamps are logged. These informations as well as the motion logged by the optical tracking system are then used as input for the 4D dose calculation.

Both approaches will be experimentally validated in this work.

\subsection{The deforming grid $4 D$ dose calculation}

As described above, the 4DDC algorithm used at our institute (Boye et al. (2013)) is based on a deforming dose grid to model the motion of the patient. To obtain this information, deformable image registration is first used to estimate the motion fields between each phase of the 4DCT (or 4DMRI) and the reference CT (usually the end of exhalation phase). These motion fields are then used to deform the dose calculation grid as a function of time. Although the motion still needs to be extracted from 4D imaging (with inevitably limited temporal resolution), using a deforming grid, it is straightforward and computationally efficient to improve the temporal resolution of the motion by simply interpolating to any defined interval between the phases of the original imaging data set. As such, this 4D dose calculation approach can operate with temporal resolutions at the millisecond level. For all validations in this work, a single field of a three field plan calculated for a liver tumour with a CTV volume of 403cc has been used. The field was optimised on the end-exhale phase of the 4DCT and prescribed a physical dose of $0.6 \mathrm{~Gy}$. This corresponds to the dose for a single field of a three field plan delivering 2.0Gy(RBE) to the tumour, assuming an RBE of 1.1 (Paganetti (2014)).

\subsection{Retrospective $4 D$ dose calculation}

Previous studies have shown the potential sensitivity of $4 \mathrm{D}$ dose calculations to not only variations in the motion (see below), but also to exact knowledge of the delivery dynamics (e.g. delivery time-line and exact starting phase, see e.g. Bernatowicz et al. (2013), Zhang et al. (2012)). Therefore, in order to be able to validate the calculation against measurement as accurately as possible, the time and length of the delivery of each pencil beam needs to be precisely known. As such, in order to obtain the most accurate information about delivery dynamics, so-called retrospective $4 \mathrm{D}$ dose distributions, calculated using information extracted from delivery log files generated at 


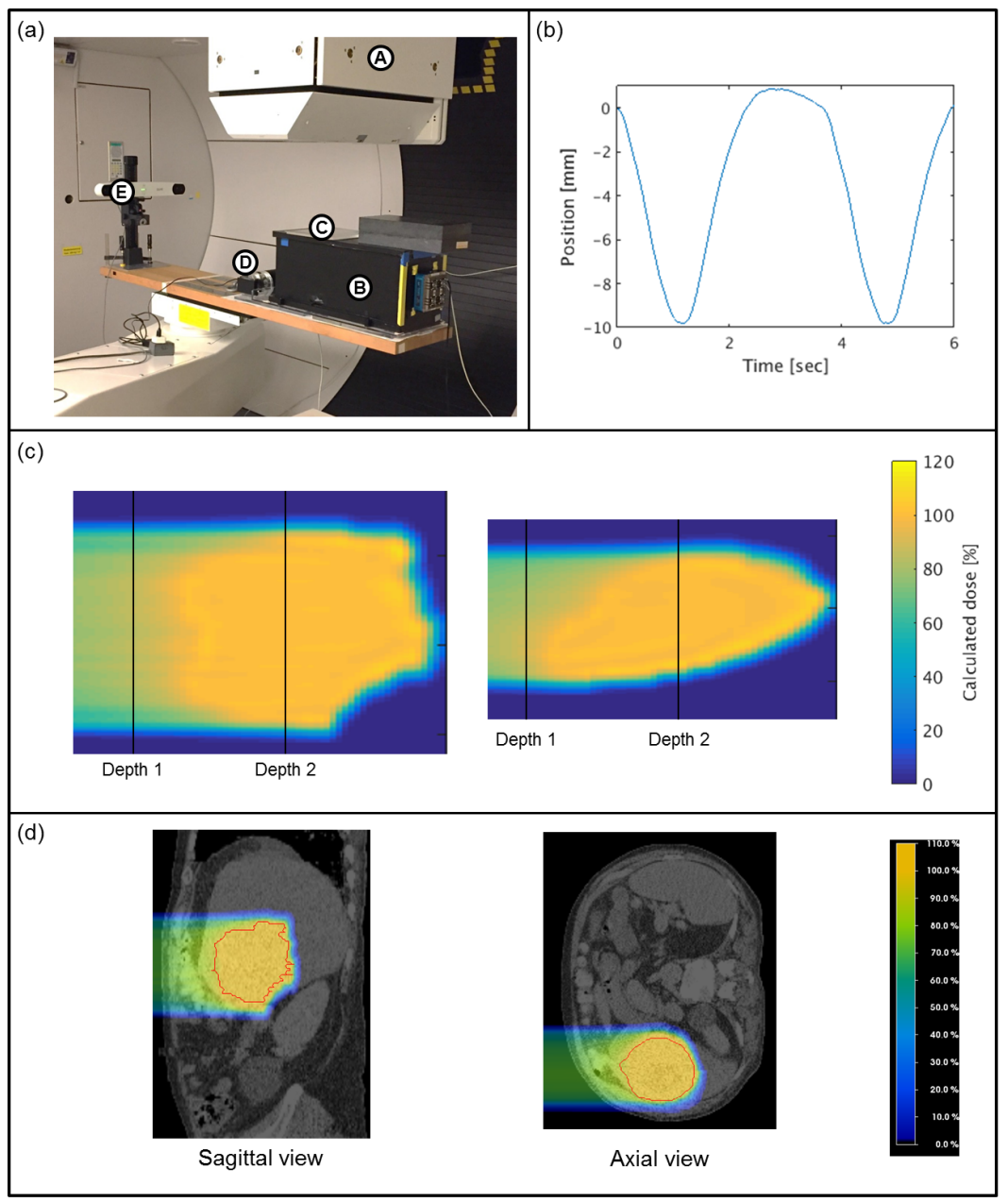

Figure 2: (a) Depiction of the measurement setup: $A$ Gantry nozzle; $B$ scintillating CCD system; $C$ PMMA phantom; $D$ Quasar phantom; $E$ Optical tracking system. (b) Illustration of the motion pattern. (c) Indication of the measurement depths, shown on the central slice of the static dose distribution (calculated in water) in both scanning directions. (d) Same slices as in (c), calculated in the original patient geometry.

the time of each spot delivery (see e.g. Meier et al. (2015), Scandurra et al. (2016) and Belosi et al. (2017)), have been calculated using a high resolution dose calculation grid of $1 \mathrm{mmx} 1 \mathrm{~mm}$ as well as with the clinically used resolution of $4 \mathrm{mmx} 4 \mathrm{~mm}$ in the plane orthogonal to the applied field, and a slice thicknesss of $2.4 \mathrm{~mm}$ (perpendicular to the applied field). In addition, the exact motion pattern, as recorded by an optical tracking system (see Section 2.4), was used as an input for the calculations. As such, these calculations are based on the most accurate temporal information for both delivery and phantom motion. This procedure is depicted in Figure 1. 


\subsection{Prospective $4 D$ dose calculation}

Making use of accurately logged delivery and motion information for the 4D dose calculation provides the best experimental conditions for verifying the accuracy of the calculation. However, in clinical practice, when 4DDCs are used to predict the best motion mitigation strategy or even for use in a 4D optimisation procedure (see Graeff et al. (2012), Bernatowicz et al. (2017)), the true motion and delivery dynamics will not necessarily be known a priori. As such, for prospective $4 \mathrm{D}$ calculations (i.e. to be performed before treatment delivery) these need to be estimated from parametrisations of the performance of the scanning system (scanning speed, average beam intensity, energy change time, to name a few parameters), together with an assumption on the motion of the patient on the day of treatment. In order to estimate the effect of such assumptions, prospective 4D dose calculations have also been performed as a comparison. Here, the information of the spot positions and weights have been extracted from the machine steering file (rather than the log file), using standardised estimates of the delivery dead times and beam intensities of the PSI Gantry 2. Such estimates do not take into account the inevitable small variations in the machine performance on the day of the delivery however. In addition, the motion of the platform was predicted assuming perfectly repeatable breathing cycles based on a single breathing cycle extracted from a 4DCT, without any variations in period and amplitude that are inevitable in the actual experiment (and in the respiration of a patient). These prospective calculations thus represent the performance and meaningfulness of the dose calculation as an evaluation tool for predicting the effects of target motion. For the prospective calculations, a dose calculation grid size of $4 \mathrm{mmx} 4 \mathrm{~mm}$ was used, being typical for clinical dose calculations at our institute, and reflecting more the dose calculation resolution that would be used routinely, also for 4D dose calculations.

\subsection{Experimental measurement setup and procedures}

All validation measurements were performed on Gantry 2 at PSI (Pedroni et al. (2004)) using a sliding motion platform (4D Quasar ${ }^{\circledR}$ phantom, Modus Medical Devices, London, Ontario, Canada) on which a scintillating-CCD dosimeter (as described in Schaetti et al. (2013)) was placed. The setup is shown in Figure 2(a). Such a detection device, although relative in nature, provides very high spatial resolution measurements $(0.3 \mathrm{~mm})$ which, given that even small motions can lead to quite localised dose distribution changes with a resolution finer than that of the pencil beam size or spacing (Phillips et al. (1992)), we believe is important for such validation measurements. To perform measurements at two different depths through the field, homogeneous sheets of PMMA were placed directly on the scintillating foil of the CCD system. The depths are shown schematically in Figure 2(c) with the corresponding water equivalent depths given in Table 1. All 4D measurements were performed using a 1-D motion pattern of a tumour extracted from a 4DCT from another patient with the same motion pattern being repeated for the duration of the delivery (see Figure $2(\mathrm{~b})$ ). Thus all measurements 
Experimental validation of a deforming grid $4 D$ dose calculation for PBS proton therapy 7

\begin{tabular}{lllcc} 
Scans & Amplitude & Period & Depth 1 & Depth 2 \\
\hline Single & $0 \mathrm{~mm}$ & - & $5.2 \mathrm{~cm}$ & $11.1 \mathrm{~cm}$ \\
Single & $10 \mathrm{~mm}$ & $3.7 \mathrm{~s}$ & $5.2 \mathrm{~cm}$ & $11.1 \mathrm{~cm}$ \\
6x Layered & $10 \mathrm{~mm}$ & $3.7 \mathrm{~s}$ & $5.2 \mathrm{~cm}$ & $11.1 \mathrm{~cm}$ \\
6x Volumetric & $10 \mathrm{~mm}$ & $3.7 \mathrm{~s}$ & $5.2 \mathrm{~cm}$ & $11.1 \mathrm{~cm}$ \\
6x Volumetric & $10 \mathrm{~mm}$ & $4.4 \mathrm{~s}$ & $5.2 \mathrm{~cm}$ & $11.1 \mathrm{~cm}$
\end{tabular}

Table 1: Overview over the measured scenarios. The motion pattern was the same for all scenarios, but with different periods. The depths are given as water equivalence.

were performed using a reproducible breathing cycle. The motion had an amplitude of $10.68 \mathrm{~mm}$ (SD: $0.09 \mathrm{~mm}$ ) and was parallel to the craniocaudal axis of the patient and to the slower lateral scanning direction of Gantry 2. 4D dose distributions for two different motion periods (3.7s and $4.4 \mathrm{~s}$ ) were measured, as was the distribution for the statically applied field. These periods have been selected such as not to be integer multiples of each other (which can lead to similar interplay effects), in order to have quite different motion effects in the calculated and measured distributions. Finally, single scan, 6x layered and 6x volumetric deliveries of the field have all been applied and analysed. For both, a hybrid rescanning technique was used, where for each spot, the dose per scan was divided by the total number of scans, but with the number of re-scans being successively reduced for low weighted spots. This approach is described fully in Zhang et al. (2015). Thus, in total, 10 combinations of motion, delivery, and depth have been delivered and measured, as summarised in Table 1. For all measurements, the delivery log files were saved and used as input to the retrospective 4DDC as described above. Additionally, the position of the measurement device was accurately logged using an optical tracking system (OTS) with a temporal resolution of 60Hz (Fattori et al. (2017)). The deliveries were triggered using the gating function of the OTS, ensuring that every delivery started at the same motion phase and allowing for synchronisation of delivery and motion. There was a delay of roughly $18 \mathrm{~ms}$, induced by the processing of the logged motion. The motion data were used as input, together with the delivery log file, for the retrospective $4 \mathrm{DDC}$ to provide the most accurate information of the actual temporal dynamics of the delivered field and platform motion.

\subsection{Data analysis}

For data analysis, all calculated dose distributions were interpolated linearly in the measurement plane to match the spatial resolution of the CCD camera $(0.313 \mathrm{mmx} 0.313 \mathrm{~mm})$. All pixels with a dose higher than $5 \%$ of the maximal calculated dose in the corresponding dose slice were chosen as analysis region. The two corresponding distributions were shifted in order to match the lateral fall-offs of the central line profiles in both directions. As in this work we are performing relative dosimetry (rather than absolute) the measured CCD intensity distributions were 
then scaled in a way such that the dose-difference-histogram with the corresponding calculated distribution peaked at zero. No quenching correction has been used. Two dimensional gamma analyses $(3 \% / 3 \mathrm{~mm}, 1 \% / 3 \mathrm{~mm}, 3 \% / 1 \mathrm{~mm}$, and $1 \% / 1 \mathrm{~mm})$ were performed between the measurements and the retrospectively calculated dose distributions to quantify the quality of agreement for both resolutions used, and to also investigate whether differences in the distributions originate more from relative dose differences or from spatial disagreement. Furthermore, dose-area-histograms were used to compare the homogeneity of the measured and calculated dose distributions and absolute dose-difference-histograms were calculated to estimate the level of agreement. A similar approach was used for the prospective calculations, but only for the coarser resolution used $(4 \mathrm{mmx} 4 \mathrm{~mm})$.

\section{Results}

\subsection{Comparison of $4 D$ dose distributions between retrospective calculation and} measurement

3.1.1. Dose distributions and dose-area-histograms The comparison of the measured and the calculated dose distributions are shown in Figure 3 . The measurement depths are indicated on top, as shown in Figure 2(c). Measured intensities are in the top row, calculated dose distributions are in the bottom row (both with the original spatial resolution). The comparisons show excellent visual agreement for all 10 scenarios (static doses are not shown here). The good agreement is also supported by the dosearea-histograms shown in Figure 4 for the case of unmitigated motion. Dashed lines correspond to the motion scenarios, while the solid lines represent the corresponding histograms for the static case as a reference. It can be seen that the histograms of the measurement and the calculation match equally well for both static and motion case. The slopes of the motion cases are less steep than for the static case which indicates a lower homogeneity, which is also seen by visual comparison.

3.1.2. 2D gamma analysis To quantify the $2 \mathrm{D}$ agreement, gamma analysis with criteria $3 \%$ dose difference / $3 \mathrm{~mm}$ distance to agreement was applied and the corresponding gamma scores are given in Table 2. For all scenarios, the agreement between calculation and measurement is higher than 95\% in the analysed region (see Section 2.5). The average gamma score over all measurements is as high as $99.22 \%$, with a standard deviation of $0.96 \%$. For the three scenarios with mitigated motion, the $2 \mathrm{D}$ gamma agreements are comparable to the unmitigated motion scenarios. The agreement of the distal measurement depth is on average $1.2 \%$ lower than for the more proximal one. Figure 5 shows the comparison of different gamma criteria for one example case (unmitigated motion at depth 2). The corresponding pass rates are given in this figure as well. It is seen that the gamma score for $1 \% / 3 \mathrm{~mm}$ is comparable to the one for criterion $3 \% / 1 \mathrm{~mm}$, which indicates that the relative dose agreement is equally good as 

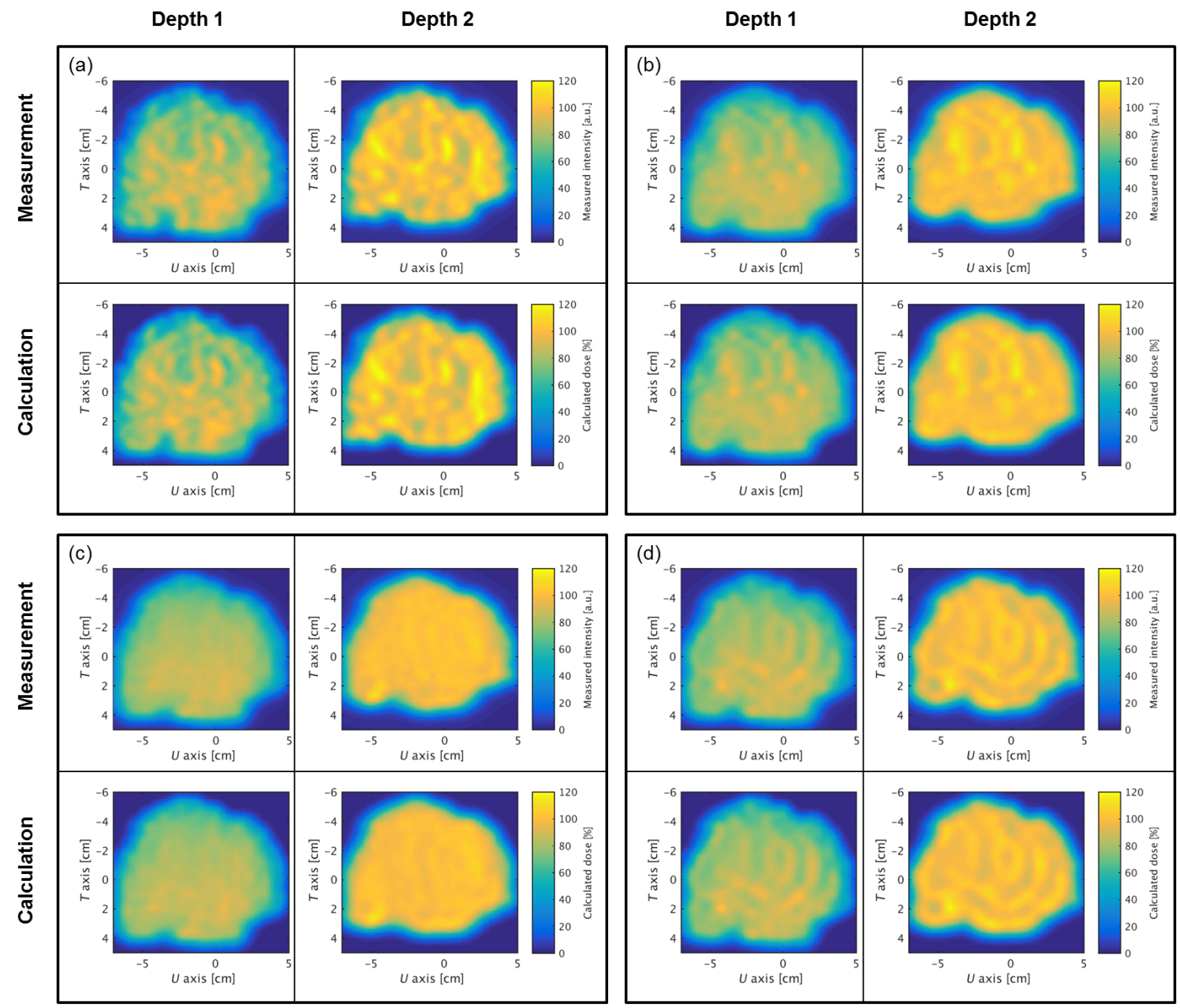

Figure 3: Comparison of the measurements and the calculations for all measured depths, indicated on top (see Figure 2(c)). (a) Unmitigated motion, period; 3.7s (b) 6x layered rescanning, period; 3.7s; (c) $6 \mathrm{x}$ volumetric rescanning, period; $3.7 \mathrm{~s}$; (d) 6x volumetric rescanning, period; $4.4 \mathrm{~s}$.

the spatial agreement.

3.1.3. Relative dose differences and dose-difference-histograms The pixel-wise absolute differences between the scaled measurements and retrospective calculations were also calculated. The resulting dose-difference-histograms are summarised in Figure 6. For each scenario, the corresponding histogram is covered by the blue band, whereas the solid line represents the median frequency for each percentage. The right hand side of Figure 6 shows the percentages of the analysed areas where the absolute dose difference exceeds $5 \%$ of the maximal calculated dose. 


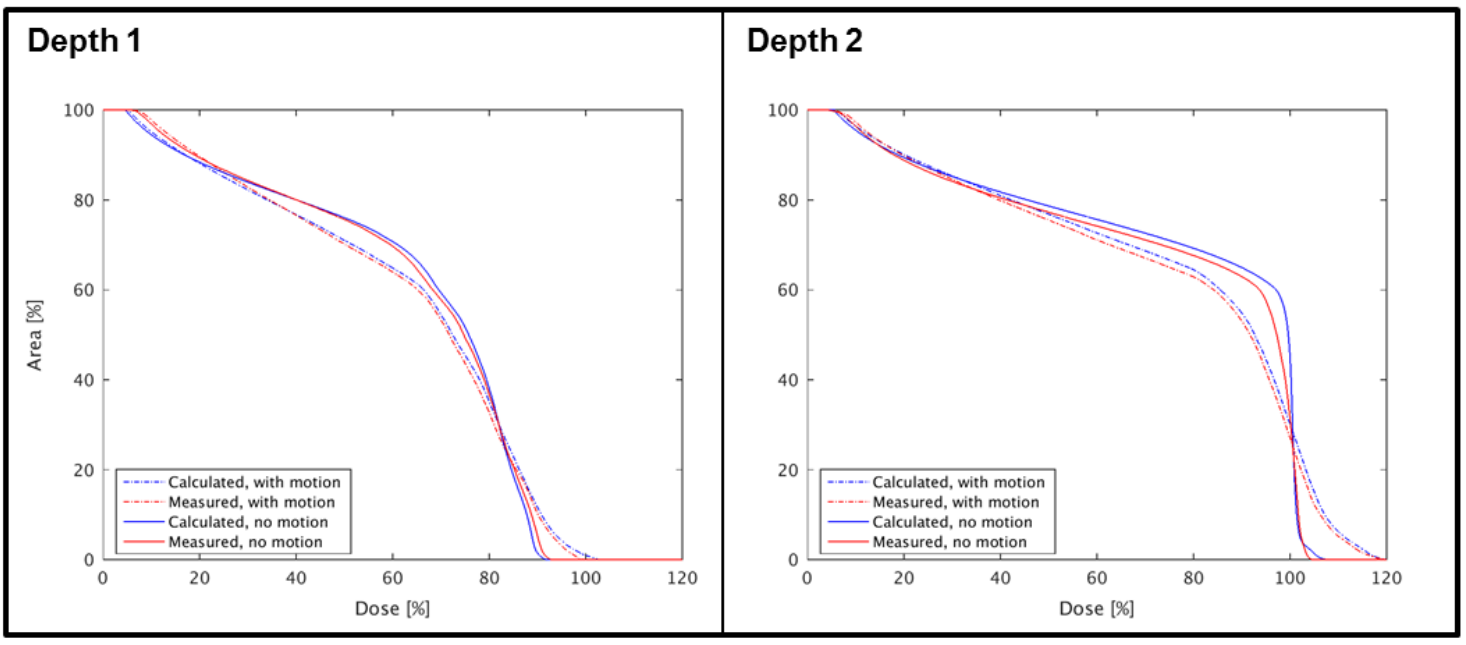

Figure 4: Dose-area-histograms for the unmitigated motion case for the proximal (left) and the distal (right) depth. The total area includes all pixels with more than $5 \%$ of calculated maximum dose. The solid lines show the dose-area-histogram of the corresponding static dose distributions as a reference.

\begin{tabular}{l|ll|l} 
3\%/3mm & Depth 1 & Depth 2 & Mean \\
\hline No motion & $99.98 \%$ & $96.88 \%$ & $98.43 \%$ \\
NR, fast motion & $99.18 \%$ & $98.29 \%$ & $98.74 \%$ \\
LR, fast motion & $99.99 \%$ & $99.35 \%$ & $99.67 \%$ \\
VR, fast motion & $99.98 \%$ & $99.80 \%$ & $99.89 \%$ \\
VR, slow motion & $99.98 \%$ & $98.81 \%$ & $99.40 \%$ \\
\hline Mean & $99.82 \%$ & $98.63 \%$ & $\mathbf{9 9 . 2 2 \%}$
\end{tabular}

Table 2: Summary of the gamma scores with criteria $3 \% / 3 \mathrm{~mm}$ for all scenarios. The numbers in the top row correspond to the measurement depths as indicated in Figure 2(c). NR: no rescanning, LR: 6x layered rescanning, VR: $6 \mathrm{x}$ volumetric rescanning, fast motion: 10mm amplitude and $3.7 \mathrm{~s}$ period, slow motion: $10 \mathrm{~mm}$ amplitude and $4.4 \mathrm{~s}$ period.

\subsection{Comparison of $4 D$ dose distributions between prospective and retrospective dose calculation}

When comparing the prospective and retrospective motion patterns, a $0.4 \mathrm{~s}$ time shift was found between the repeated motion patterns (from 4DCT motion estimation) and the actually logged motion patterns at the end of the delivery. This time shift was the result of inevitable uncertainties in the platform motion and the conversion of the motion to the control system of the motion platform. The gamma comparisons in the upper row of Figure 7 show that the impact of this motion shift on the $4 \mathrm{D}$ dose distribution is rather small, especially for the short dose delivery using a single scan, where the shift in motion was smaller. The difference in gamma pass rate is $2 \%$ for the estimated and also 


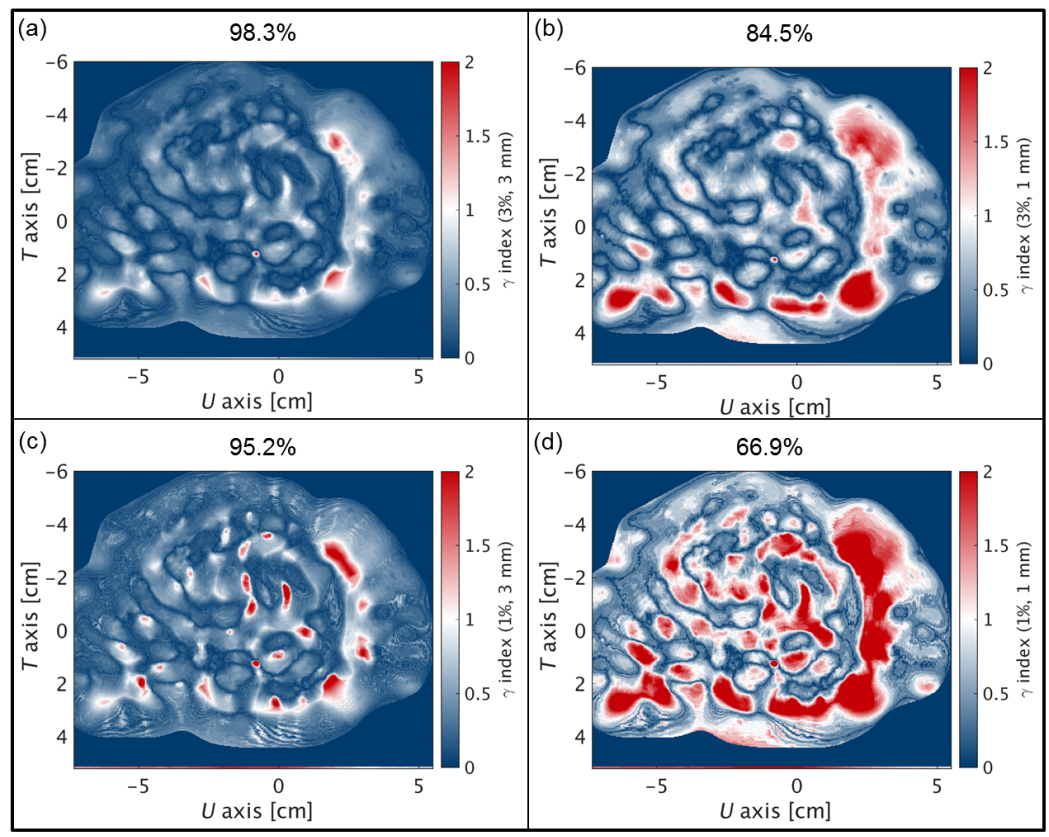

Figure 5: Comparison of the gamma maps with various criteria, given for the single scan delivery (unmitigated) in presence of motion at mid-SOBP. The numbers on top indicate the gamma pass rates. Gamma criteria: (a) $3 \% / 3 \mathrm{~mm}$, (b) $3 \% / 1 \mathrm{~mm}$, (c) $1 \% / 3 \mathrm{~mm}$, (d) $1 \% / 1 \mathrm{~mm}$.

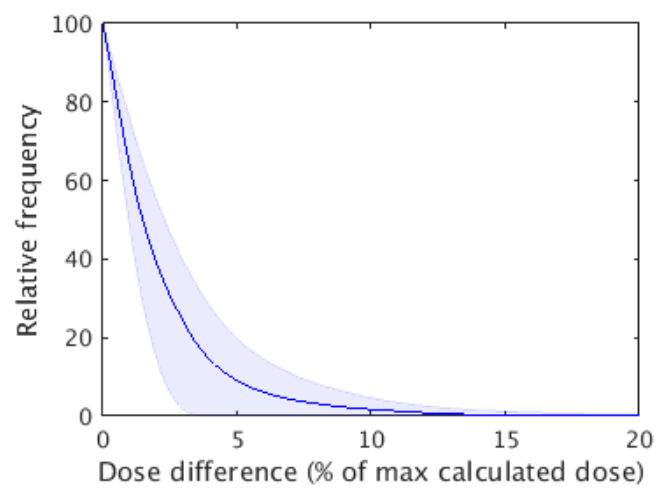

\begin{tabular}{l|ll} 
& Depth 1 & Depth 2 \\
\hline No motion & $3.09 \%$ & $9.14 \%$ \\
NR, fast motion & $5.44 \%$ & $9.82 \%$ \\
LR, fast motion & $2.65 \%$ & $8.08 \%$ \\
VR, fast motion & $2.60 \%$ & $9.27 \%$ \\
VR, slow motion & $2.93 \%$ & $8.99 \%$ \\
\hline Average: & \multicolumn{2}{|c}{$\mathbf{6 . 2 \%}$}
\end{tabular}

Figure 6: Left: range of absolute dose differences for all scenarios, in percent of the maximal calculated dose of the respective scenario. Solid line: median values. Right: Summary of the percentages of the area with more than 5\% (of the maximal calculated dose) absolute dose difference in the analysed region.

for the actual time stamps in Figure 7(a), and the pattern of the gamma maps is similar as well. On the other hand, when moving to estimates of the time stamps, rather than the more exact time stamps extracted from the log-file, a much higher impact on the agreement with the measurement was found, as shown in the lower row of Figure 7(a). The gamma pass rates drop by $24 \%$ when exchanging the actual spot time stamps with the estimated ones. However, it is seen that the errors originating from the estimated time stamps seem to be smeared out for rescanned deliveries (Figure $7(\mathrm{~b})$ ). Gamma 

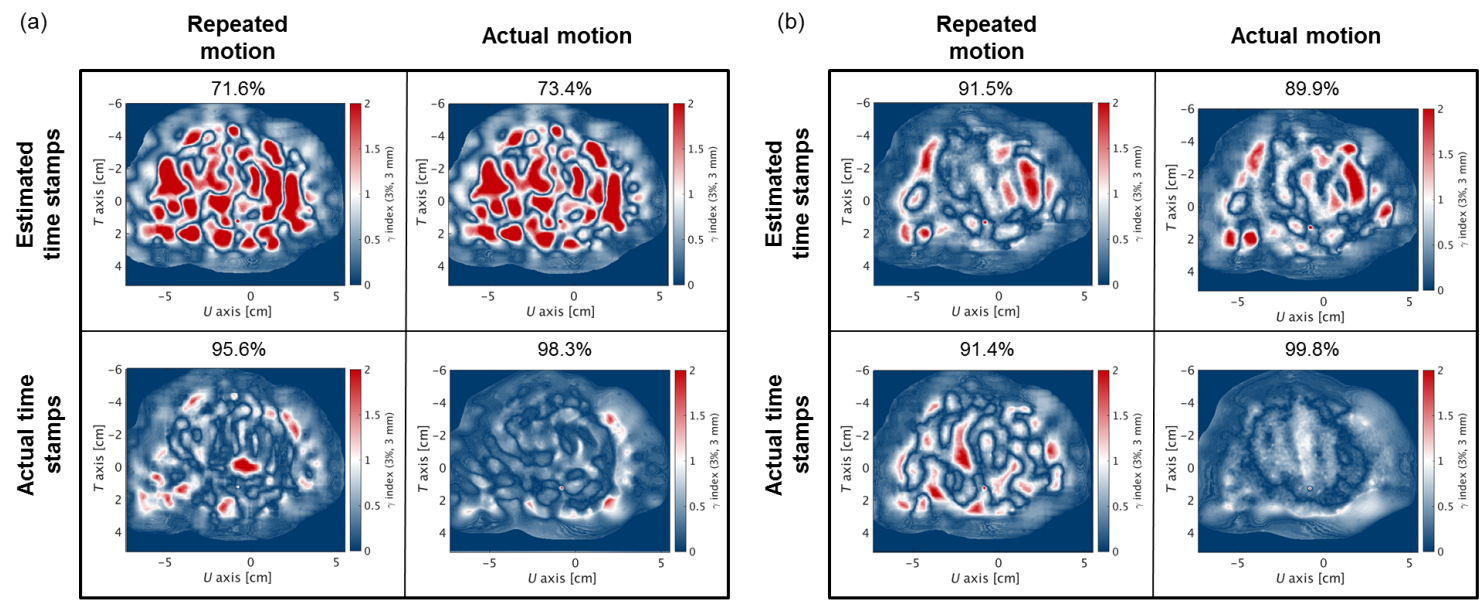

Figure 7: Gamma maps for different inputs to the 4DDC, with indicated gamma pass rates. (a) Unmitigated scenario, (b) volumetric rescanning (both 3.7s period). Top row: time stamps as estimated by the machine control file, bottom row: actual time stamps from the delivery $\log$ files. Left: the first motion cycle of the sliding platform is repeated, right: the actual (complete) logged motion is given as input.

scores for all cases in this figure are given in Figure 7.

\section{Discussion}

In this study, we have systematically compared measured and calculated 4D dose distributions to verify the accuracy of the deforming grid $4 \mathrm{D}$ dose calculation. In order to validate the algorithm as accurately as possible, for the retrospective case, the most accurate estimates of the temporal dynamics of both the delivery and motion have been used, extracted from delivery log files and direct motion monitoring of the motion platform respectively. Under these conditions, excellent agreement between 4D measurement and calculation have been achieved, validating the accuracy of the temporal modelling of motion by the deforming grid 4D dose calculation. One needs to keep in mind however that this assumption is not fulfilled in clinical conditions, where specifically the motion and the density changes of the patients are highly variable and only known to a limited extent. Nevertheless, it is important to know how accurately a calculation tool can predict outcomes under the given conditions. Furthermore, rescanning seems to smear out the effects of the variable motion patterns and time stamps, meaning that even in clinical conditions, the 4DDC can give a reasonable estimation of the effectiveness of different motion mitigation techniques.

The $4 \mathrm{D}$ dose calculations agreed very well with the corresponding measurements in many of the delivery scenarios investigated in this study using different comparison metrics. However, there were remaining imprecisions which may have several origins. Probably the most influential one is the quenching which the measurements were not corrected for. It is known that the light output of a scintillating screen is quenched 
when irradiated, most dominantly in the Bragg peak region. This may explain the lower gamma agreements in the distal measurement depth for most scenarios, since the used measurement device includes such a scintillator. It is seen in Figure 2(c) that for some lateral positions, depth 2 is towards the distal end of the field, which means that there are certainly some highly weighted Bragg peaks placed in the measured region. In order to understand the effect of the linear interpolation of the dose distribution to the resolution of the measurements, the gamma analysis was additionally performed directly on the original calculation resolution. This had no significant impact on the gamma pass rates. Nevertheless, although the resolution of the $4 \mathrm{D}$ dose distributions is more coarse than the measurement resolution, it is still useful to have highly resolved measurements, since our algorithm calculates point doses (Phillips et al. (1992)) and no resolution limit is given for the deformation of the calculation grid. Like this, even small deviations in position can be captured. Also, the retrospective dose distributions have been additionally calculated on the clinical calculation grid (with a resolution of $4 \mathrm{mmx} 4 \mathrm{~mm}$ ), with only minimal changes in the gamma pass rates. The corresponding data can be found in the appendix. As the aim of this work was to validate the temporal accuracy of the 4D dose calculation, only one dimensional motion, without any range variations or deformations, has been considered. This has been done deliberately in order to have exact control over the motions used for the validation and, given that the $4 \mathrm{D}$ dose calculation is fully symmetric in the plane perpendicular to delivery, a validation of the temporal accuracy of the approach in one direction is also a validation of its accuracy on the other. For the case of density heterogeneities, the 4D dose algorithm uses an identical approach as used by our 3D clinical algorithm, which has been extensively validated for the static case by Albertini et al. (2011). Nevertheless, we aim to extend this validation using a more complex phantom that includes both density heterogeneities and deformable motions in the future. A first work in this direction has been performed by Perrin et al. (2017) using a customised anthropomorphic phantom.

Nevertheless, the in general very good agreement found in this study opens the opportunity to reconstruct 4D dose distribution after each delivery. This technique can be used to assure an accurate treatment delivery, and if necessary to re-optimise the treatment plan for compensating the inhomogeneities in the previously delivered fractions. This reconstruction method represents reality even better when using rescanning, which will be the primary motion mitigation technique used at our institute. When the exact motion of a patient is known, the delivered dose distribution can therefore be precisely known.

This study also showed that uncertainties in the delivery timeline can have a big impact on the resulting 4D dose distribution. For sophisticated 4D treatments like 4D optimisation (Bernatowicz et al. (2017)), a more accurate prediction for the spot timing is therefore critical. One possibility to reach this goal is to retrospectively analyse the machine log files from past deliveries as additional QA for the 4DDC. Since many patients have been treated with our Gantry 2, and many experiments using rescanned fields have been performed, all the corresponding log files could create a data base for 
a statistical analysis of the delivery time lines under many different conditions. This is expected to lead to better predictions of prospective 4D dose distributions.

\section{Conclusion}

We have demonstrated that the deforming grid 4D dose calculation can predict the complex patterns of $4 \mathrm{D}$ dose distributions due to the interplay effect with high dosimetric accuracy. Even in the presence of motion or timing uncertainties, it is a valid clinical tool for the selection of patient specific motion mitigation techniques, 4D treatment planning in general, and eventually for 4D optimisation and tracking. Furthermore, this work confirms the validity of all the studies that have already been performed or will be performed in the future using this calculation tool.

\section{Acknowledgments}

This work was mainly funded by the Swiss National Science Foundation (SNF) grant No. 320030_163330/1 (MK). Partial funding was additionally received from the European Community's Seventh Framework Programme (FP7/2007-2013) under grant agreement No. 290605 (PSI-FELLOW/COFUND) (GF) and from the Giuliana and Giorgio Stefanini Foundation (GK). 
Experimental validation of a deforming grid $4 D$ dose calculation for PBS proton therapy 15

\section{References}

Albertini F, Casiraghi M, Lorentini S, Rombi B \& Lomax A J 2011 Experimental verification of IMPT treatment plans in an anthropomorphic phantom in the presence of delivery uncertainties Phys. Med. Biol. 56, 4415-4431.

Ammazzalorso F \& Jelen U 2014 A 4D dose computation method to investigate motion interplay effect in scanned ion beam prostate therapy Phys. Med. Biol. 59, N91-N99.

Belosi M F, van der Meer R, de Acilu Laa P G, Bolsi A, Weber D C \& Lomax A J 2017 Treatment $\log$ files as a tool to identify treatment plan sensitivity to inaccuracies in scanned proton beam delivery Radiotherapy and Oncology Article in press.

Bernatowicz K, Lomax A J \& Knopf A 2013 Comparative study of layered and volumetric rescanning for different scanning speeds of proton beam in liver patients Phys. Med. Biol. 58, 7905-7920.

Bernatowicz K, Peroni M, Perrin R, Weber D C \& Lomax A J 2016 Four-dimensional dose reconstruction for scanned proton therapy using liver 4DCT-MRI Int. J. Radiation Oncology Biol. Phys. 95(1), 216-223.

Bernatowicz K, Zhang Y, Perrin R, Weber D C \& Lomax A J 2017 Advanced treatment planning using direct 4D optimisation for pencil-beam scanned particle therapy Phys. Med. Biol. (accepted for publication).

Bert C \& Durante M 2011 Motion in radiotherapy: particle therapy Phys. Med. Biol. 56, R113-R144.

Bert C, Groezinger S O \& Rietzel E 2008 Quantification of interplay effects of scanned particle beams and moving targets Phys. Med. Biol. 53, 2253-2265.

Bert C \& Rietzel E 2007 4D treatment planning for scanned ion beams Radiation Oncology 2:24.

Boye D, Lomax A J \& Knopf A C 2013 Mapping motion from 4D-MRI to 3D-CT for use in 4D dose calculations: A technical feasibility study Med. Phys. 40(6), 061702-1 - 061702-11.

Dueck J, Knopf A C, Lomax A J, Albertini F, Persson G F, Josipovic M, Aznar M, Weber D C \& af Rosenschold P M 2016 Robustness of the voluntary breath-hold approach for the treatment of peripheral lung tumors using hypofractionated pencil beam scanning proton therapy Int. J. Radiation Oncology Biol. Phys. 95(1), 534-541.

Engelsman M, Schwarz M \& Dong L 2013 Physics controversies in proton therapy Seminars in Radiation Oncology 23(2), 88-96.

Engwall E, Hynning E, Janssens G \& Glimelius L 2016 Proceedings to the 55 Annual Meeting for the Particle Therapy Cooperative Group (PTCOG) Int. J. Particle Therapy: Summer 2016 3(1), 71-276.

Fattori G, Safai S, Carmona P F, Peroni M, Perrin R, Weber D C \& Lomax A J 2017 Monitoring of breathing motion in image-guided PBS proton therapy: comparative analysis of optical and electromagnetic technologies Radiation Oncology 12:63.

Gorgisyan J, Perrin R, Lomax A J, Persson G F, Josipovic M, Engelhom S A, Weber D C \& af Rosenschold P M 2017 Impact of beam angle choice on pencil beam scanning breath-hold proton therapy for lung lesions Acta Oncologica 56(6), 853-859.

Graeff C, Durante M \& Bert C 2012 Motion mitigation in intensity modulated particle therapy by internal target volumes covering range changes Med. Phys. 39(10), 6004-6013.

Groezinger S O, Bert C, Kraft G \& Rietzel E 2008 Motion compensation with a scanned ion beam: a technical feasibility study Radiation Oncology 3, 34 .

Kang Y, Zhang X, Chang J Y, Wang H, Wei X, Liao Z et al. 2005 4D proton treatment planning strategy for mobile lung tumors Int. J. Radiation Oncology Biol. Phys. 67, 906-914.

Knopf A C, Bert C, Heath E, Nill S, Kraus K, Richter D, Hug E, Safai S, Albertini F, Zenklusen S, Boye D, Soukup M, Soehn M, Sobotta B \& Lomax A J 2010 Special report: Workshop on 4D-treatment planning in actively scanned particle therapy - recommendations, technical challenges, and future research directions Med. Phys. 37, 4608-4614.

Knopf A C, Boye D, Lomax A J \& Mori S 2013 Adequate margin definition for scanned particle therapy in the incidence of intrafractional motion Phys. Med. Biol. 58(17), 6079-6094. 
Knopf A C, Hong T S \& Lomax A J 2011 Scanned proton radiotherapy for mobile targets - the effectiveness of re-scanning in the context of different planning approaches and for different motion characteristics Phys. Med. Biol. 56(22), 7257-7271.

Li Y, Kardar L, Li H, Cao W, Chang J Y et al. 2014 On the interplay effects with proton scanning beams in stage III lung cancer Med. Phys. 41(2), 021721.

Meier G, Besson R, Nanz A, Safai S \& Lomax A J 2015 Independent dose calculations for commissioning, quality assurance and dose reconstruction of PBS proton therapy Phys. Med. Biol. 60, 28192836 .

Paganetti H 2014 Relative biological effectiveness (RBE) values for proton beam therapy. Variations as a function of biological endpoint, dose, and linear energy transfer Phys. Med. Biol. 59, R419R472.

Paganetti H, Jiang H \& Trofimov A 2005 4D Monte Carlo simulation of proton beam scanning: modelling of variations in time and space to study the interplay between scanning pattern and time-dependent patient geometry Phys. Med. Biol. 50, 983-990.

Pedroni E, Bearpark R, Boehringer T, Coray A, Duppich J, Forss S, George D, Grossmann M, Goitein G, Hilbes C, Jermann M, Lind S, Lomax A J, Negrazus M, Schippers M \& Kotrle G 2004 The PSI Gantry 2: a second generation proton scanning gantry Z. Med. Phys. 14, 25-34.

Perrin R L, Zakova M, Peroni M, Bernatowicz K, Bikis C, Knopf A C, Safai S, Fernandez-Carmona P, Tscharner N, Weber D C, Parkel T C \& Lomax A J 2017 An anthropomorphic breathing phantom of the thorax for testing new motion mitigation techniques for pencil beam scanning proton therapy Phys. Med. Biol. 62.

Pfeiler T, Baumer C, Engwall E, Spaan B \& Timmermann B 2017 4D dose computation in pencil beam scanning proton therapy has been clinically implemented and experimentally verified Presented at the 56 Annual Meeting for the Particle Therapy Cooperative Group (PTCOG) .

Phillips M H, Pedroni E, Blattmann H, Boehringer T, Coray A \& Scheib S 1992 Effects of respiratory motion on dose uniformity with a charged particle scanning method Phys. Med. Biol. 37(1), 223234.

Richter D, Saito N, ad M Haertig N C, Ellerbrock M, Jaekel O, Combs S E, Habermehl D, Herfarth K, Durante M \& Bert C 2014 Four-dimensional patient dose reconstruction for scanned ion beam therapy of moving liver tumors Int. J. Radiation Oncology Biol. Phys. 89(1), 175-181.

Richter D, Schwarzkopf A, Trautmann J, Kraemer M, Durante M, Jaekel O \& Bert C 2013 Upgrade and benchmarking of a 4D treatment planning system for scanned ion beam therapy Med. Phys. 40(5), 051722-1 - 051722-17.

Rietzel E, Chen G T Y, Choi N C \& Willet C G 2005 Four-dimensional image-based treatment planning: target volume segmentation and dose calculation in the presence of respiratory motion Int. J. Radiation Oncology Biol. Phys. 61, 1535-1550.

Scandurra D, Albertini F, van der Meer R, Meier G, Weber D C, Bolsi A \& Lomax A J 2016 Assessing the quality of proton PBS treatment delivery using machine log files: comprehensive analysis of clinial treatments delivered at PSI Gantry 2 Phys. Med. Biol. 61, 1171-1181.

Schaetti A, Zakova M, Meer D \& Lomax A J 2013 Experimental verification of motion mitigation of discrete proton spot scanning by re-scanning Phys. Med. Biol. 58, 8555-8572.

Testa M, Schumann J, Lu H M, Shin J, Faddegon B, Perl J \& Paganetti H 2013 Experimental validation of the TOPAS Monte Carlo system for passive scattering proton therapy Med. Phys. 40(12), 121719-1 - 121719-16.

Zhang Y, Boye D, Tanner C, Lomax A J \& Knopf A C 2012 Respiratory liver motion estimation and its effect on scanned proton beam therapy Phys. Med. Biol. 57, 1779-1795.

Zhang Y, Huth I, Wegner M, Weber D C \& Lomax A J 2016 An evaluation of rescanning technique for liver tumour treatments using a commercial PBS proton therapy system Radiation and oncology 121(2), 281-287.

Zhang Y, Huth I, Wegner M, Weber D C \& Lomax A J 2017 Surface as motion surrogate for gated re-scanned pencil beam proton therapy Phys. Med. Biol. 62, 4046. 
Zhang Y, Knopf A C, Tanner C, Boye D \& Lomax A J 2013 Deformable motion reconstruction for scanned proton beam therapy using on-line X-ray imaging Phys. Med. Biol. 58, 8621.

Zhang Y, Knopf A C, Tanner C \& Lomax A J 2014 Online image guided tumour tracking with scanned proton beams: a comprehensive simulation study Phys. Med. Biol. 59, 7793.

Zhang Y, Knopf A C, Weber D C \& Lomax A J 2015 Improving 4D plan quality for PBS-based liver tumour treatments by combining online image guided beam gating with rescanning Phys. Med. Biol. 60, 8141. 


\section{Appendix A. Dose calculations on a more coarse grid}

The retrospective dose distributions have been additionally calculated on the clinical

calculation grid with a resolution of $4 \mathrm{mmx} 4 \mathrm{~mm}$. The corresponding gamma pass rates can be found in Table A1.

\begin{tabular}{l|ll|l}
$\mathbf{3 \%} / \mathbf{3 m m}$ & Depth 1 & Depth 2 & Mean \\
\hline No motion & $99.91 \%$ & $97.20 \%$ & $98.56 \%$ \\
NR, fast motion & $98.23 \%$ & $97.87 \%$ & $98.05 \%$ \\
LR, fast motion & $99.98 \%$ & $99.32 \%$ & $99.65 \%$ \\
VR, fast motion & $99.98 \%$ & $99.52 \%$ & $99.75 \%$ \\
VR, slow motion & $99.98 \%$ & $98.81 \%$ & $99.40 \%$ \\
\hline Mean & $99.62 \%$ & $98.54 \%$ & $\mathbf{9 9 . 0 8 \%}$
\end{tabular}

Table A1: Summary of the gamma scores with criteria $3 \% / 3 \mathrm{~mm}$ for all scenarios, calculated on the coarse calculation grid. The numbers in the top row correspond to the measurement depths as indicated in Fig. 2(c). NR: no rescanning, LR: 6x layered rescanning, VR: 6x volumetric rescanning, fast motion: $10 \mathrm{~mm}$ amplitude and $3.7 \mathrm{~s}$ period, slow motion: $10 \mathrm{~mm}$ amplitude and $4.4 \mathrm{~s}$ period. 